\title{
Imaging Rough Paper to Evaluate Methods for Soot Removal
}

\section{Teresa T. Duncan, Edward P. Vicenzi, ${ }^{*}$ and Shannon A. Brogdon-Grantham}

Museum Conservation Institute, Smithsonian Institution, Washington, DC

*vicenzie@si.edu

\begin{abstract}
Here, we use a variety of microscopic imaging techniques, including scanning electron microscopy, 3D visible light microscopy, and portable microscopy to capture the topography of rough paper. Paper coated with a model soot was cleaned with either a firm vinyl eraser or softer, putty-like kneaded eraser and reimaged to characterize the disposition of remaining soot. Although both methods remove soot from the upper-most surface of the paper, only the kneaded eraser can conform to the complex topography to remove soot from the interstices between paper fibers.
\end{abstract}

Keywords: paper, soot, cleaning, cultural heritage, roughness

\section{Introduction}

Soot is notoriously challenging to remove from surfaces, owing to its nanoscale size and low polarity $[1,2]$. In cases where soot is deposited on rough or porous surfaces, for example, fire damaged materials, the challenge of soot removal for conservators of cultural heritage increases in complexity: soot located on the upper-most features of rough topographies can more easily be removed than soot that has collected within deeper parts of the substrate structure. Although firm materials, such as vinyl erasers, can be quite effective at cleaning surface features, the inability to conform to complex topographies makes cleaning rough surfaces difficult (Figures 1a, 1b, 1d). However, a soft, deformable material, such as a putty-like kneaded eraser, can conform to rough surfaces and is therefore capable of removing soot from crevices within the structure (Figures 1a, 1c, 1e). Earlier work [3,4] has shown how soft, elastic materials can be used to remove particulate contaminants from patterned model surfaces, but here we examine paper that has a much more complex surface topography.

By imaging a soot-deposited rough paper after using different cleaning materials, we aim to better understand how the physical properties of cleaning materials can be leveraged for efficient soot removal. The first goal of this work was to collect images of a pristine paper surface to document its topographic complexity. Although we used laboratory-based techniques, including scanning electron microscopy (SEM) and $3 \mathrm{D}$ visible light microscopy, to image the paper, we also show how a low-cost portable microscope can be used to capture the sought-after information. The second goal of this work was to apply these methodologies to image papers that have been coated with soot and subsequently cleaned. Microscopic examination of the paper substrates after cleaning provides an in-depth understanding into each method's success for the removal of soot.

\section{Methods and Materials}

Materials. Rough, gelatin-sized watercolor paper (Arches) with a reported paper weight of 300 grams per meter was used as the substrate. Lamp black pigment (Natural Pigments), a white vinyl eraser (Magic Rub \#1954, Prismacolor), and a kneaded eraser (Prismacolor) were used as received.

Sample preparation. To prepare the pristine paper sample, a $2 \mathrm{~cm} \times 1 \mathrm{~cm}$ section was cut and mounted on a glass slide with double-sided tape. To prepare two cleaned samples, samples were first coated (while working outdoors at $11^{\circ} \mathrm{C}$ and $28 \%$ humidity) with lamp black pigment, a soot commercially prepared from burning oil, and then cleaned. Specifically, masking tape was used to adhere $8.3 \times 8.3 \mathrm{~cm}$ samples of paper to the inner bottom of a plastic container, $0.2 \mathrm{~g}$ of lamp black pigment was sprinkled on the surface of the paper, the lid was secured, and the container was shaken for $30 \mathrm{sec}$. After allowing to rest for $2 \mathrm{~min}$, the masking tape was removed from the samples, a Giotto Rocket Blower was used to remove loosely adhered pigment, and samples were cut to $2 \mathrm{~cm} \times 1 \mathrm{~cm}$ and mounted on glass slides with doublesided tape. A white vinyl eraser was cut into $1 \mathrm{~cm}$ cubes with an $\mathrm{X}$-acto knife, and these small pieces were tamped on half of the surface of one coated sample. A portion of the kneaded eraser was kneaded by hand for $\sim 20 \mathrm{sec}$ and then tamped on

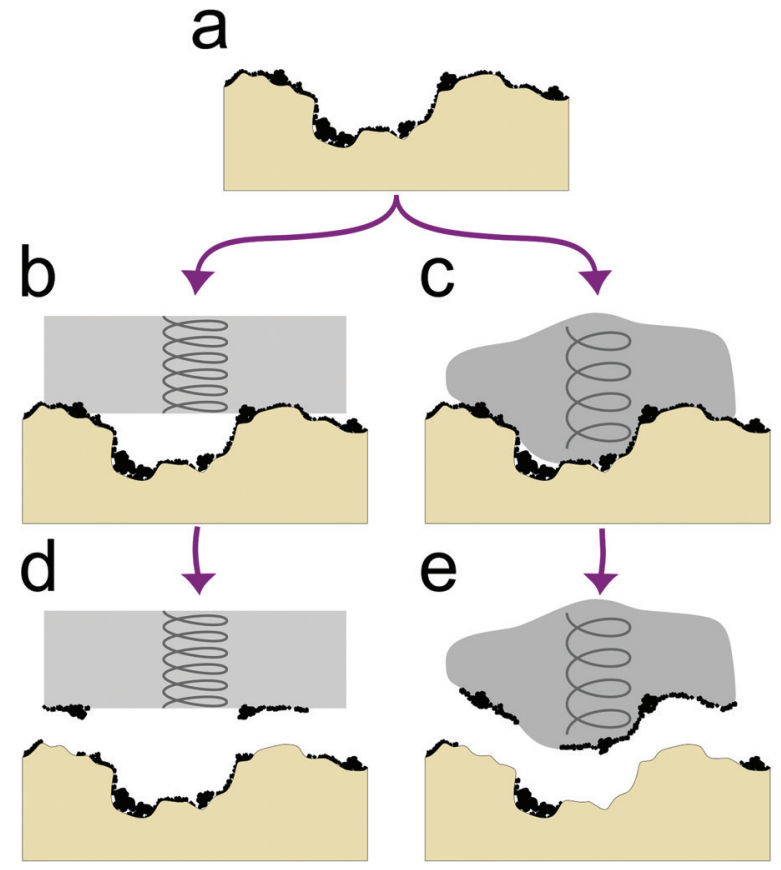

Figure 1: A soot-coated paper surface (a) can be cleaned with various types of materials, ranging from stiff elastomers (b), which typically contact only the surface-most features of a rough surface, to deformable putties (c), which can conform to rough topographies. In this work, we imaged surfaces after cleaning with either a stiff vinyl eraser (d) or a softer, putty-like kneaded eraser (e) to explore how the ability of cleaning materials to conform to rough surfaces affects cleaning. The tension of the spring represents the stiffness of the material. 


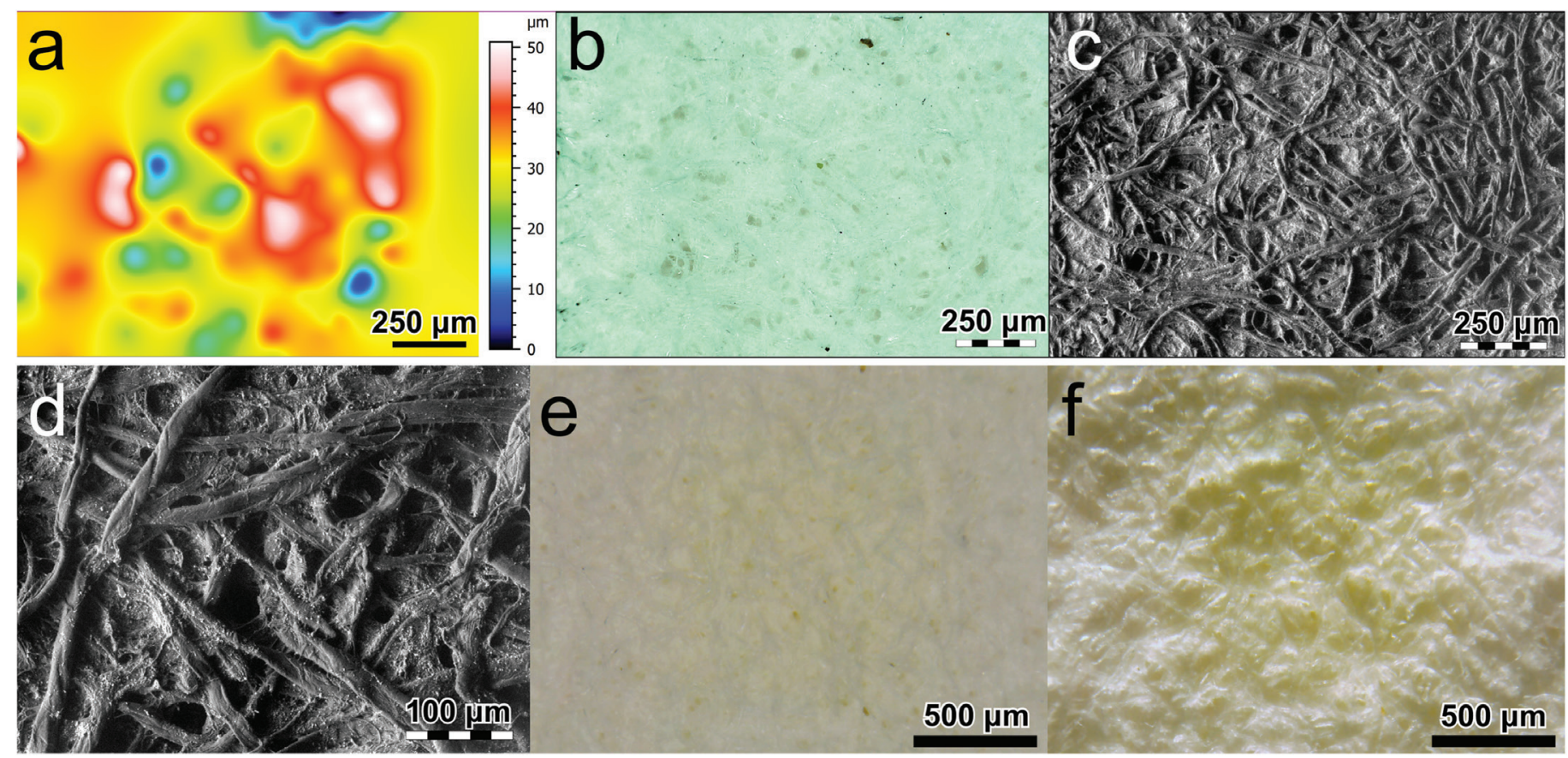

Figure 2: Images of pristine (that is, with no soot deposition) paper captured with a 3D visible light microscope (a, b), SEM (c, d), and a portable microscope under normal light (e) or raking light (f) illumination.

half of the surface of the other coated sample. Cleaning was performed until pigment was no longer noted visually on the cleaning materials.

Portable light microscopy. Samples were imaged with an 8MP Qscope digital USB microscope (Model QS.8.200P). The built-in LED ring-lights were used to collect normal illumination reflected light images. For raking light images, a gooseneck LED light source set at approximately $20^{\circ}$ was used to illuminate the surface. Images were flat field-corrected using ImageJ [5] and the Calculator Plus plug-in [6], which was used to divide the original image by a background image that consisted of a blurred image of the lightest gray square (Neutral 8) of an Xrite ColorChecker target. A Gaussian blur was applied to the background image using ImageJ to blur surface features of the target surface as a substitute to using an out-of-focus image, because changing the focus on the Q-scope microscope also changes the field-of-view for a given working distance.

3D visible light microscopy. A Hirox KH-8700 digital microscope was used to collect 2D and 3D images of the paper surface. Focal stacks were comprised of between 220-250 images with a Z-axis step size of $750 \mathrm{~nm}$ between images. The 3D images were processed using Digital Surf MountainsLab ${ }^{\circledR}$ v9 software to determine roughness (Sq) after leveling, following procedures defined in ISO 25178 [7].

Scanning electron microscopy. A Hitachi S3700N SEM was used to collect images in variable pressure mode (100 Pa ambient atmosphere) via a Gatan DigiScan ${ }^{\mathrm{TM}}$ II system and GMS v3 software. Segments of a 5-segment backscattered electron (BSE) detector were collected for each region imaged, with the off-optic axis E-segment used to best depict topographic relief of the paper surface.
Correlation of white light $3 \mathrm{D}$ visible light and SEM images. Rough alignment between the two imaging methods was aided by placing $\mathrm{Cu}$ tape fiducial markers adjacent to the cut edge of the paper. Fine-scale registration was then performed using MountainsLab ${ }^{\circledR} \mathrm{v} 9$ to overlay images at the fiber length scale. Mountains v9 was additionally used to produce several $3 \mathrm{D}$ images collected in the SEM using BSE detector segments.

\section{Results}

The pristine paper was imaged with several techniques to capture the surface morphology prior to soot deposition. The surface roughness of the paper was determined from the root mean square height (Sq) to be $6.5 \pm 0.8 \mu \mathrm{m}$ from $3 \mathrm{D}$ visible light microscopy, with the surface map and associated focal stack white light images shown in Figures 2a and 2b, respectively. Although the surface map does not easily resolve the individual fibers, the dimensions of these fibers were determined from SEM images (Figures 2c, 2d) to be $15.9 \pm 5.5 \mu \mathrm{m}(\mathrm{n}=67)$ in width. The portable microscope was used to capture images under normal and raking light: the normal light illumination (Figure 2e) appears to visually "flatten" the surface, whereas the raking light illumination showcases the rough topography of the paper (Figure 2f).

After coating the rough paper with soot and cleaning with either a vinyl eraser or kneaded eraser (Figures $3 b$ and $3 c$, respectively), the visual appearance of the paper was significantly improved but not returned to the pristine appearance (Figure 3a). From 3D visible light microscopy (Figures $4 \mathrm{a}, 4 \mathrm{~b}$ and $4 \mathrm{f}, 4 \mathrm{~g}$ ), Sq was measured for the papers cleaned with either a vinyl eraser or kneaded eraser as $9.9 \pm 0.6 \mu \mathrm{m}$ and $11.6 \pm 1.3 \mu \mathrm{m}$, respectively (Figure 6). Fiber width was measured from SEM images (Figures $4 \mathrm{c}$ and $4 \mathrm{~h})$ to be $18.7 \pm 5.4 \mu \mathrm{m}(\mathrm{n}=42)$ and $17.3 \pm 4.6 \mu \mathrm{m}(\mathrm{n}=60)$, respectively. The location of soot within the paper topography 


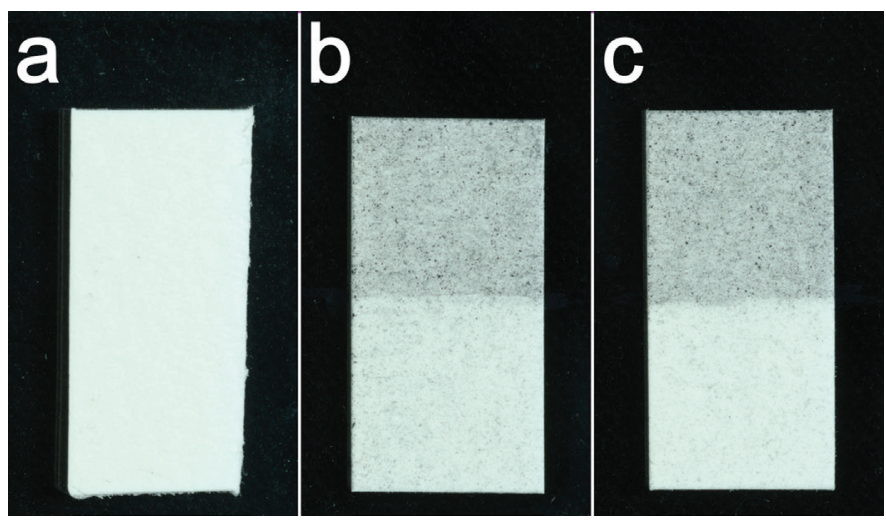

Figure 3: Photographs of paper samples before (a) and after coating with soot and cleaning the lower half of the paper sample with a vinyl eraser (b) and a kneaded eraser (c). Paper samples are approximately $2.5 \mathrm{~cm} \times 1.3 \mathrm{~cm}$.

can be examined by overlaying correlated images collected with SEM and 3D visible light microscope using white light (Figures $4 \mathrm{~b}$ and $4 \mathrm{c}$; Figures $4 \mathrm{~g}$ and $4 \mathrm{~h}$ ). The images collected with the portable microscope under normal and raking light show high contrast between the soot and paper, also allowing for a visual assessment of how effectively soot is removed from the entire surface (Figures $4 \mathrm{~d}$ and $4 \mathrm{i}$ ) and where soot is located within the surface topography (Figures $4 \mathrm{e}$ and $4 \mathrm{j}$ ).

\section{Discussion}

Paper, in general, is composed of a network of randomly oriented fibers (for example, cellulose) typically embedded with fillers (for example, chalk) to modify surface absorbency [8-10]. The artists' watercolor paper we have employed in this study is also externally sized with gelatin, meaning a thin layer of gelatin has been applied to the surface to decrease surface wettability and protect the surface [11]. The resulting surface topography is a complex mesh of overlapping fibers with pits and crevices between fibers that can be especially difficult to clean. An ideal cleaning treatment should be able to effectively remove contaminants from the surface without disrupting paper fibers or filler particles.
The microscopic examination of paper substrates before and after cleaning methods conducted here explored three questions: 1) How do the physical properties of cleaning materials influence the location of residual soot on the substrate? 2) How do the topographic features of the paper substrate dictate the efficacy of each method? 3) What physical changes occur to the substrate with cleaning? The images collected with the portable optical microscope provide a wealth of information regarding the cleaning process. Images collected under normal illumination display high contrast between the soot particles and the paper substrate, allowing for the overall efficacy to be assessed by an evaluation of the image histogram (described in a forthcoming study). Although both cleaned samples are coated with a fine dispersion of soot particulates, the kneaded eraser (Figure 4i) was more effective than the vinyl eraser (Figure $4 \mathrm{~d}$ ) in the removal of larger soot aggregates. Images collected under raking light show where, within the paper topography, these larger aggregates are located, with large aggregates appearing in darker, and therefore deeper, areas of the paper. These soot aggregates appear to have been removed from the paper cleaned with the kneaded eraser, suggesting that the kneaded eraser could conform to the paper topography to enable contact with the soot aggregates located in deeper portions of the paper structure.

The disposition of soot aggregates within the paper structure was also observed through the overlay of correlated images collected using SEM and white light microscopy. This analysis confirms that soot aggregates tend to be located deeper within the paper structure. Although this information is similar to that provided by the portable microscope, the SEM images offer improved fidelity of cellulose fibers, coupled with the ability to localize soot aggregates using quantified height information in the $3 \mathrm{D}$ images.

Microscopy was also used to investigate the presence of any physical changes (such as damaged fibers or filler removed from the surface) that may be caused by the cleaning methods used here. Portable microscopy was not as useful, as the magnification required to assess these types of changes was not sufficiently high; however, SEM does attain the required level of magnification and detail. Because BSE images reveal
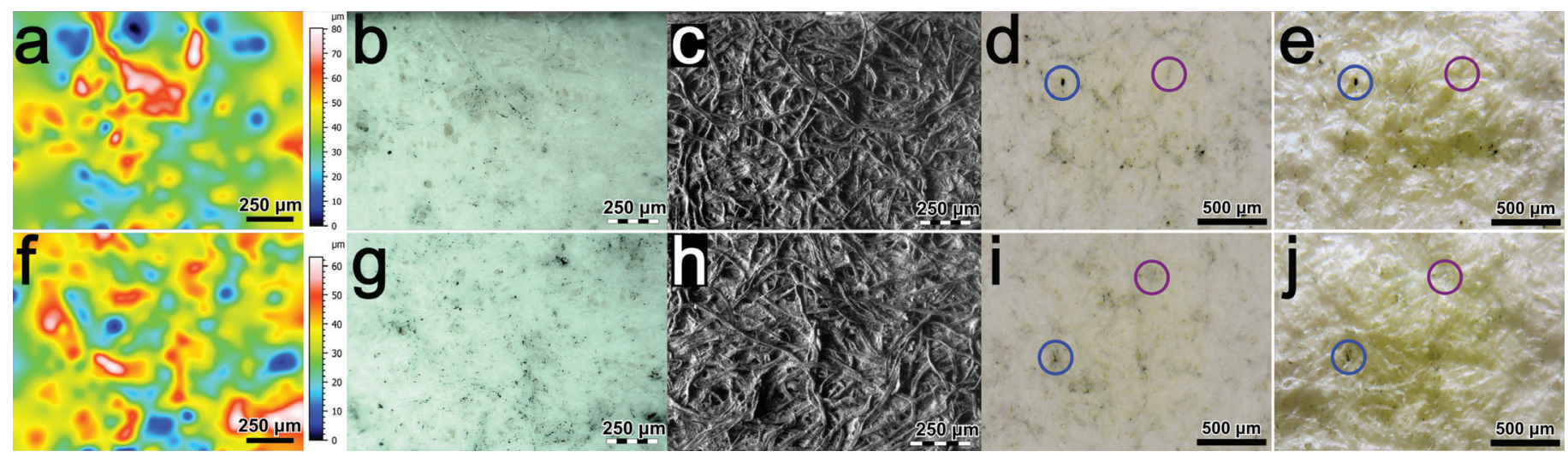

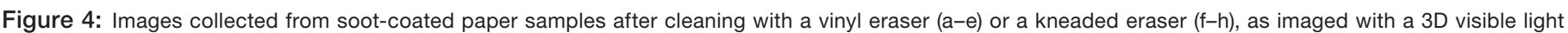

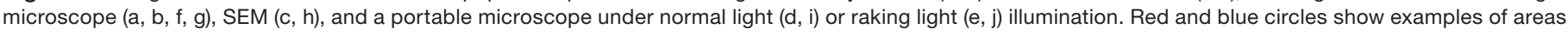
with finely dispersed soot and large aggregates, respectively. 


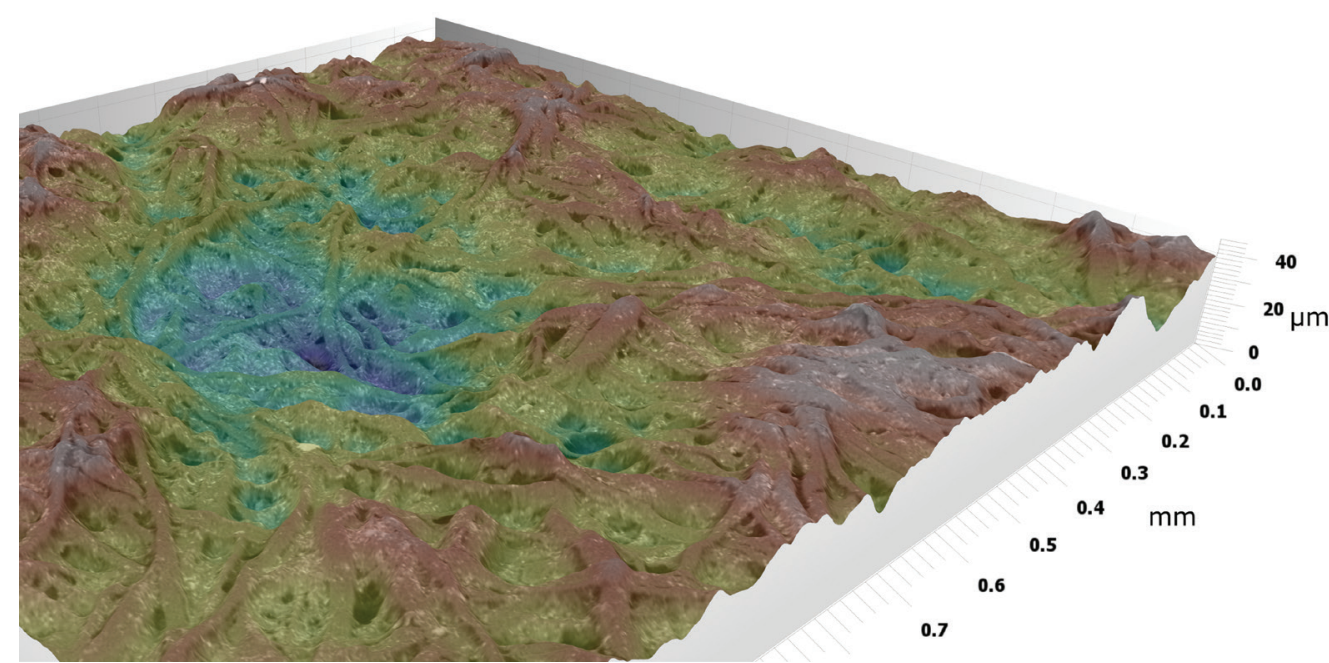

Figure 5: Oblique view of pristine rough paper with a false colored (rainbow) look-up table used to depict elevation differences in the SEM image of the surface.

required to design new materials for cleaning applications.

\section{Conclusion}

The different imaging modalities used for this study work in concert to detail which methods are the most successful for removing soot from paper and to provide insight into how these methods function. Specifically, we have been able to show how soft, deformable materials like putties can conform to rough surfaces for more effective cleaning. Firm cleaning materials like vinyl erasers can be very effective for cleaning relatively flat areas of paper. However, they are less effective for the removal of particles resting against paper fibers and located deeper within pits and along high angle surfaces.

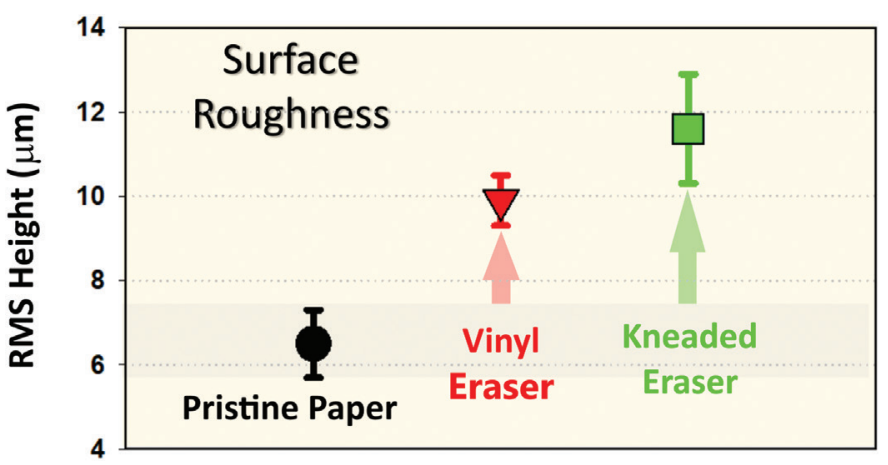

Figure 6: Surface roughness $(\mathrm{Sq})$ of paper obtained by 3D digital light microscopy before and after tamping with either a vinyl or kneaded eraser.

high contrast between paper fibers and filler particulates, these components can be differentiated from each other and compared before and after cleaning.

From the images collected (Figures $4 \mathrm{c}$ and $4 \mathrm{~h}$ ), we see no apparent indications of damage to paper fibers or reduction in the distribution of filler particulates using the gentle tamping motions employed in our cleaning protocol. An oblique view of the pristine rough paper surface illustrates the scale of the initial topography (Figure 5). However, an increase in roughness as determined by $3 \mathrm{D}$ visible light microscopy (Figure 6) for both cleaning treatments indicates that the surface has changed. We attribute the larger change in roughness after tamping with the kneaded eraser to the tackiness of the material, which previously has been shown to pull fibers from a paper surface [12].

Although two cleaning materials are showcased in this report, we are currently engaged in a study of a larger array of cleaning materials with various physical attributes to correlate certain physical properties of the cleaning materials with cleaning efficacy. We hope our studies will aid paper conservators in the selection of cleaning materials for specific use cases and provide experimental researchers with the information
Although tacky materials (for example, kneaded erasers) are very effective for the removal of soot particles from the paper substrate, care must be taken with their application to the cleaning of paper, as their use may be inappropriate on delicate papers.

\section{Acknowledgements}

T.D. acknowledges the Smithsonian Postgraduate/Postdoctoral Fellowship in Conservation of Museum Collections for funding this research project. E.P.V. is grateful to Digital Surf for providing extended at-home licensing during the COVID-19 pandemic.

\section{References}

[1] D Bolstad-Johnson, AIC News 35 (2010) 2-5.

[2] S Spafford-Ricci and F Graham, J Amer Inst Conservation 39 (2000) https://doi.org/10.1179/019713600806113310.

[3] TT Duncan et al., ACS Appl Mater Interfaces 11 (2019) https://doi.org/10.1021/acsami.9b17602.

[4] TT Duncan et al., J Cultural Heritage 48 (2021) https://doi .org/10.1016/j.culher.2020.11.004.

[5] CA Schneider et al., Nat Methods 9 (2012) https://doi .org/10.1038/nmeth.2089.

[6] W Rasband and G Landini, Calculator Plus, (2009) https:// imagej.nih.gov/ij/plugins/calculator-plus.html.

[7] ISO 25178-2:2012, https://www.iso.org/standard/42785 .html.

[8] MB Shaw and MJ O'Leary, J Res Natl Bureau Standards 21 (1938) https://doi.org/10.6028/jres.021.037.

[9] A Dwan, J Amer Inst Conservation 26 (1987) https://doi .org/10.1179/019713687806027924.

[10] D Van Der Reyden, J Amer Inst Conservation 31 (1992) https://doi.org/10.1179/019713692806156394.

[11] T Barrett and C Mosier, J Amer Inst Conservation 34 (1995) https://doi.org/10.1179/019713695806124620.

[12] EJ Pearlstein et al., J Amer Inst Conservation 22 (1982) https://doi.org/10.1179/019713682806028496. 